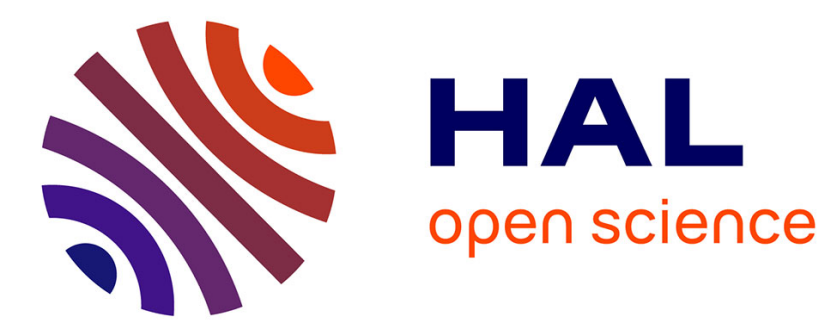

\title{
Enhancement and performance analysis of channel access mechanisms in Terahertz band
}

Lina Aliouat, Mohammed Rahmani, Hakim Mabed, Julien Bourgeois

\section{To cite this version:}

Lina Aliouat, Mohammed Rahmani, Hakim Mabed, Julien Bourgeois. Enhancement and performance analysis of channel access mechanisms in Terahertz band. Nano Communication Networks, 2021, 29, pp.100364 (14). hal-03549345

\section{HAL Id: hal-03549345 \\ https://hal.science/hal-03549345}

Submitted on 31 Jan 2022

HAL is a multi-disciplinary open access archive for the deposit and dissemination of scientific research documents, whether they are published or not. The documents may come from teaching and research institutions in France or abroad, or from public or private research centers.
L'archive ouverte pluridisciplinaire HAL, est destinée au dépôt et à la diffusion de documents scientifiques de niveau recherche, publiés ou non, émanant des établissements d'enseignement et de recherche français ou étrangers, des laboratoires publics ou privés. 


\title{
Enhancement and Performance Analysis of Channel Access Mechanisms in Terahertz Band
}

\author{
Lina Aliouat ${ }^{\mathrm{a}, *}$, Mohammed Rahmani $^{\mathrm{b}}$, Hakim Mabed $^{\mathrm{a}}$, Julien Bourgeois $^{\mathrm{a}}$ \\ ${ }^{a}$ University Bourgogne Franche-Comté, FEMTO-ST Institute, CNRS, Montbéliard, France \\ ${ }^{b}$ Huazhong University of Science and Technology, Hubei Engineering Research Center on Big Data Security, Wuhan, China
}

\begin{abstract}
With Terahertz band $(0.1-10 \mathrm{THz})$ technology, several nanoscale ad hoc applications could be developed, such as intrabody health monitoring, programmable material, etc. The fundamental element to ensure the functionality of these applications is the way to access the radio communication channel. The main challenge with nanocommunication is the need to efficiently share the terahertz radio spectrum to optimize data transmission in nanonetworks. In this context, Spread in Time On-Off Keying (TS-OOK) protocol was proposed as a channel access mechanism that uses Femtosecond-Long pulse-based OOK modulation. Furthermore, different new improvements of TS-OOK were introduced, such as RD-TSOOK and SRHTSOOK approaches.

In this paper, we review and compare the proposed OOK-based channel access techniques with our new approach called SDMA-TSOOK. The design of the proposed scheme takes into account the weaknesses of these mechanisms in terms of channel access balancing, co-channel collision, and networking capacity that pose a problem for concurrent channel access. We evaluate the performance of our proposed protocol using the BitSimulator. The obtained results showed that SDMA-TSOOK ensures 100\% equitable access between nanodevices, as well as a better success rate with an insignificant number of collisions as compared to the published reference protocols.
\end{abstract}

Keywords: Nanonetworks, Terahertz band, Channel access, Mac layer, Femtosecond-long pulse

\section{Introduction}

In recent years, nanotechnology has continually provided a new set of tools to design nanoscale devices called nanodevices. Due to their constrained size, nanodevices are characterized by their severe limitations in computational capacity, memory storage, energy source, etc. Thus, these nanodevices can only perform simple specific functionality such as sensing, identification, and computing [1]. However, by collaboration via nanonetworking, a large number of nanodevices enable achieving valuable application goals. These applications, running on nanonetworks, may concern several areas such as biomedical [2] (e.g., monitoring

\footnotetext{
${ }^{*}$ Corresponding author

Email addresses: lina.aliouat@univ-fcomte.fr (Lina Aliouat), i201922029@hust.edu.cn (Mohammed Rahmani), hmabed@femto-st.fr (Hakim Mabed), julien.bourgeois@femto-st.fr (Julien Bourgeois)
} 
of cancer cells, glucose or cholesterol), industrial 3, 4, 5] (e.g., product quality control), environmental (e.g., potable water reservoirs monitoring) or military (e.g., surveillance against biological and chemical attacks at the nanoscale level) [6, 7], etc.

In this context, Wireless NanoNetwork (WNN) introduces a new ad hoc networking paradigm where a huge number of nanodevices densely interconnected are concentrated in a limited area. To make this paradigm effective, wireless communications technology is required to adequately ensure information exchange between nanodevices. Due to the sub-millimeter scale of the used antennas, the Terahertz frequency band seems to be the best choice to support communications for WNN applications. Indeed, the Terahertz frequency band provides more advantages such as availability of a very high channel bandwidth $(0.1-10 \mathrm{THz})$ with low energy consumption and a short communication range (much below one meter) [8]. Among others, Graphene based antennas [9, 10] are better suited to the exploitation of the Terahertz spectrum.

The convergence of the nanodevices characteristics and Terahertz frequencies prevent the use of classical communication based on frequency career signals [1, 12. Modulation and coding schema has a fundamental role in designing channel access mechanisms. Regarding the nanodevices constraints and terahertz spectrum characteristics [13, 14, narrow-band frequency modulation [15], amplitude-based modulation [16] and phasebased modulation [17, 18] are technically complex to implement. Therefore, the use of pulse-based modulation (PBM) is commonly admitted in WNNs [19, 20].

In the literature, different pulse-based modulation techniques have been proposed such as On-Off-Keying (OOK) 21] which is a particular case of Amplitude Shift Keying (ASK) where the radio pulse is transmitted only for bit 1, Pulse Amplitude Modulation (PAM), Pulse Position Modulation (PPM), and others.

In OOK modulation, energy is consumed only for transmitting the bit 1 while the bit 0 is a silence (no required energy ), which is suitable for the nanodevices' peculiarities. A bit 1 is transmitted using a 100 femtosecond-long electromagnetic pulse spread on a broad Terahertz band. OOK allows very high bit-rates and therefore very short transmission times [21].

The motivation and contributions of this paper are as follows:

- Due to the expected very high density of nanodevices in nanonetwork and the huge number of concurrent transmissions, a collisions mitigation solution is needed.

- The paper provides both an overview and comparative study of the existing OOK-based access mechanisms, then we propose a new OOK-based protocol Slot Division Multiple Access scheme, called SDMA-TSOOK, to overcome their drawbacks, in particular the lack of channel access balancing.

- The objective of the proposed protocol is to improve the performances of the channel access according to the generated interference and the equity use of the radio resources. We analytically compare our protocol to three approaches (TS-OOK, RD-TSOOK and SRH-TSOOK) on the basis of the bit collision probability and the co-channel collision probability between two active communications. We also compare the efficiency of the different studied approaches using a NanoNetwork dedicated simulator 
BitSimulator 22].

BitSimulator is a network simulator specifically targeted to electromagnetic nanonetworks. It is more accurate and suitable than other simulators like Nano-Sim (extension of the NS3 simulator) as it simulates collisions up to a bit level. Unlike Nano-Sim, BitSimulator transmits data by bits. Also, it supports very dense nanonetworks and the peculiarities of the terahertz channel. As such, it enables designing new terahertz protocols levels MAC and physical.

The rest of the paper is organized as follows: The following section introduces the motivation and related work. In Section 3, works related to channel access based OOK modulation are presented and reviewed. In Section 4, novel balanced channel access, that takes into account collisions and throughput is discussed. The details of the simulation and the performance of the proposed scheme are investigated in Section 5 . Finally, the work is concluded in Section 7 .

\section{Related Works}

The Terahertz band nanocommunications (i.e., communication among nanodevices) have constraints and requirements that significantly impact the physical layer and, hence, need an appropriate modulation and channel access mechanism [23. Due to the miniaturization of nanodevices and the peculiarities of Terahertz band technology, conventional techniques cannot directly be applied. The first reason is the bandwidth, which is limited in classical techniques, whereas the Terahertz frequency band provides almost $10 \mathrm{THz}[24$. As a second reason, classical communication is based on the transmission of continuous signals (carrier-based modulations) instead of the Terahertz band frequencies, where nanodevices cannot generate a carrier signal.

In the Terahertz band, the transmission is based on pulses spread in time exchange. Several modulation techniques and channel access solutions have been proposed in the literature for Terahertz band nanonetworks 25]. A pulse-based communication [26] is employed in the Terahertz band, which guarantees very large bitrates in the short-range. The pulse-based communication scheme is encoded either in (a) Pulse Amplitude Modulation, (b) Pulse Position Modulation, (c) Pulse Width Modulation, or (d) Pulse Rate Modulation. The peculiarities of nanodevices and the phenomena that affect the propagation of these very short pulses in the Terahertz band need a reexamination of common assumptions in pulse-based communications. So far, two alternatives of pulse-based communication, based or not on femtosecond-long pulse modulation, have drawn interest. An On-Off Keying (OOK) modulation is introduced for exchanging femtosecond-long pulses among nanodevices in [21. Some of these schemes proposed in the literature are discussed below.

The authors in 27] proposed (TH-PPM) time-hopping multiple access (THMA) with M-ary pulse position modulation (PPM) for Terahertz band based nanonetworks as a modulation and multiple access scheme for Terahertz band. In TH-PPM, the information is contained in the pulse position relative to a repetition time interval. This pulse position was defined by the time-hopping sequences (THSs) generated randomly. Note that at the reception side, due to synchronization, there could be errors that would be considered for errors in the estimated bits. 
To achieve low energy consumption in wireless nanosensor networks (WNSNs), a novel minimum energy coding scheme (MEC) is proposed in [28]. Unlike the existing nanocommunication schemes in which the whole Terahertz band is exploited, carrier frequencies are chosen among the windows in the Terahertz band. Low weight channel coding is combined with OOK modulation at every carrier frequencies to obtain efficient energy. Latency is decreased according to the lengthy codewords. Therefore, the number of multi-carrier signals should be chosen to satisfy a certain delay requirement.

In [29], authors adopted the OOK modulator and propose an optimal coding design in order to reduce transmission energy. It is focused on the design of low weight code, which translates m-bit symbols into n-bit codewords with fewer pulses. It should be noted that this work only considers the transmission energy while the encoding operation energy, conducted before transmitting the data, is not considered.

In [30, a modulation and channel sharing mechanism called Direct Sequence On-Off Keying (DS-OOK) for the Terahertz band is proposed. The direct sequence is used to distinguish concurrent transmitting. DS-OOK spreads the signal by multiplying each pulse by a user-specific spreading code. Meanwhile, its weakness rests in the fact that the receiver should know the spreading code and the expected delay.

An approach using rate division multiple access (RDMA) with on-off keying to spread very short pulses in time has been proposed in [31 for Terahertz nanonetworks. To fix the symbol interval, the authors propose a new scheme based on the Prime Mod algorithm, which generates a unique prime number at each nanodevice. For this, the scheme involved complexity cost due to the process of addition and factorization repeated until a different coprime factor is found.

The first channel access scheme pulse-based modulation in Terahertz band, called Time Spread OnOff Keying (TS-OOK), is proposed in [32. TS-OOK is based on OOK modulator. In order to mitigate interference and reduce the number of transmission errors in nanonetworks, a low-weight channel coding scheme is employed. All nanodevices use a unique symbol rate for transmitting binary streams.

In order to reduce collisions due to the unique symbol rate for all nanodevices, the authors proposed an improvement of TS-OOK in [33. The functionality of the proposal, named Rate Division Time Spread OOK (RD-TSOOK), is similar to that of TS-OOK. The main difference remains in the symbol rates that are different for different nanodevices. The lack of the protocol is the overhead introduced by the handshaking process initiated by the transmitter, which would eventually result in low channel utilization.

Another TS-OOK variant, reported in the literature, called Symbol Rate Hopping Time Spread On-Off Keying protocol (SRH-TSOOK) 34 aimed to ensure symbol rate balancing. To this, they employed pseudorandom time-hopping sequences to adjust the time between symbols that, on average, could generate similar symbol rates for nanodevices.

In [35, the authors presented a modulation scheme combining TS-OOK and the pulse position modulation (PPM). This scheme is applied in nanobody networks. Contrary to TS-OOK, which sends bits one by one, the basic idea of the proposed scheme is to transmit the bits as a sequence. However, slightly more time is needed to transmit the same bits, leading to a lower data rate than TS-OOK. 
The related work we discussed is summarized in Table 1. The following section is dedicated to the detailed description of TS-OOK, RD TSOOK and SRH-TSOOK schemes, in order to compare them with the proposal in terms of success rate and collisions.

Table 1: Summary of modulation and channel access schemes.

\begin{tabular}{|c|c|c|c|c|c|}
\hline Reference & Category & Features & $\begin{array}{c}\text { Balancing } \\
\text { access }\end{array}$ & $\begin{array}{l}\text { Complexity of } \\
\text { Transceivers }\end{array}$ & Based OOK \\
\hline 27 & $\begin{array}{l}\text { Pulse Position } \\
\text { Modulation }\end{array}$ & $\begin{array}{l}\text { PPM combined with time } \\
\text { hopping }\end{array}$ & $\mathrm{X}$ & Complex & $\mathrm{X}$ \\
\hline 28 & Pulse Coding & carrier frequencies & $\mathrm{X}$ & Complex & $\checkmark$ \\
\hline 29 & Pulse Coding & low-weight code & $\mathrm{X}$ & Complex & $\checkmark$ \\
\hline 35 & $\begin{array}{l}\text { Pulse Position } \\
\text { Modulation }\end{array}$ & PPM and time spread pulses & $\mathrm{X}$ & Complex & $\checkmark$ \\
\hline 30 & Pulse Coding & Spread codes & $\mathrm{X}$ & Complex & $\checkmark$ \\
\hline 31 & $\begin{array}{l}\text { Pulse rate } \\
\text { Modulation }\end{array}$ & $\begin{array}{l}\text { Based on TS-OOK with } \\
\text { coprime rates }\end{array}$ & $\mathrm{X}$ & Simple & $\checkmark$ \\
\hline 32 & $\begin{array}{l}\text { Pulse rate } \\
\text { Modulation }\end{array}$ & $\begin{array}{l}\text { - 100-fs pulses } \\
\text { - asynchronous transmission }\end{array}$ & $\checkmark$ & Simple & $\checkmark$ \\
\hline 33 & $\begin{array}{l}\text { Pulse rate } \\
\text { Modulation }\end{array}$ & $\begin{array}{l}\text { Combining TS-OOK and } \\
\text { coprime rates }\end{array}$ & $\mathrm{X}$ & Simple & $\checkmark$ \\
\hline 34 & $\begin{array}{l}\text { Pulse rate } \\
\text { Modulation }\end{array}$ & $\begin{array}{l}\text { Based TS-OOK with coprime } \\
\text { numbers and Simple Rate } \\
\text { Hopping }\end{array}$ & weak & Simple & $\checkmark$ \\
\hline
\end{tabular}

\section{Channel access protocols in Wireless NanoNetworks}

The nanonodes missions are carried out using communications and are constrained by the particularities of WNN, such as limited capacities of the nanonodes. Besides, communication protocols are required to regulate access to channels and coordinate the transmission between nanonodes. In this section, we will describe some of these protocols, their operating principles, advantages, and drawbacks.

\subsection{TS-OOK protocol}

Time Spread On-Off Keying is a protocol for sharing the radio channel among different nanodevices. This communication technique serves as both a modulation scheme and multiple access mechanism. TS-OOK protocol is based on the transmission of electromagnetic pulses of duration $T_{p}=100 \mathrm{fs}$ to represent a logical "1" and silence for " 0 ". The time between logical symbols $\left(T_{s}>>T_{p}\right)$ is fix for all communications. 
In Figure 1. we show an example of TS-OOK communications for the case where two nanodevices, $E_{1}$ and $E_{2}$, concurrently transmit different binary sequences to a third nanodevice $R$, such as:

- $E_{1}$ : First nanodevice transmitting the sequence "110100"

- $E_{2}$ : Second nanodevice transmitting the sequence "100101"

- $R$ : Receiver nanodevice

The second transmitter is further from the receiver than the first transmitter.

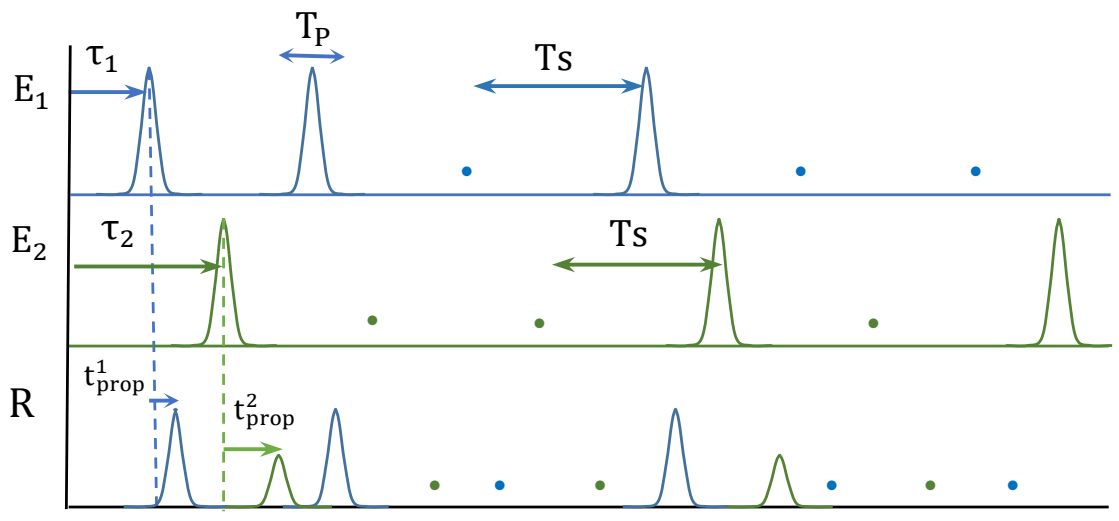

Figure 1: TS-OOK modulation: All nanodevices have the same symbol rate.

The nanodevice does not need to detect the channel continually. It merely waits for the next transmission. The transmission of logical "0" does not consume energy in emission, which guarantees better energy efficiency. The multiple access mechanism does not require a global synchronization between the nanodevices. The transmitter and receiver are synchronized thanks to the packet preamble. Indeed, the receiver detects the header of the packet throughout the transmission of a specific initial sequence. The TS-OOK modulation can support a high number of nanodevices transmitting simultaneously several gigabits per second and up to a few terabits per second, since the time between transmissions $T s$ is much longer than the pulse duration $T p$. Symbol rate $\beta$ is the ratio between $T s$ and $T p$.

$$
\beta=\frac{T s}{T p}
$$

The first drawback in TS-OOK is the complexity of finding the optimal value of the symbol rate $\beta$, in order to guarantee better flow per nanodevice according to the number of active nanodevices. In other words, if $\beta=1$, the nanodevice sends the communication symbols in burst and the maximum throughput for each nanodevice is reached. Therefore, only one nanodevice at a time can access the channel. By increasing $\beta$, the throughput per nanodevice is reduced, but the radio resources are shared over more simultaneous nanodevices. 
Secondly, in the case where two senders $T 1$ and $T 2$ start transmitting at two different times $\tau_{1}$ and $\tau_{2}$, respectively. A collision occurs between two simultaneous communications at the receiver if:

$$
\left(\tau_{1}+t 1_{\text {prop }}\right) \quad \bmod T_{s}=\left(\tau_{2}+t 2_{\text {prop }}\right) \quad \bmod T_{s}
$$

where $t 1_{\text {prop }}$ (resp. $t 2_{\text {prop }}$ ) designates the signal propagation time from $T 1$ (resp. $T 2$ ) to the receiver.

Therefore, if the transmitters and the receiver are immobile ( $t 1_{\text {prop }}$ and $t 2_{\text {prop }}$ are fixed), the collision will concern all symbols until one of the two communications is finished. The pulse collision and co-channel collision probabilities between two communications is then given by:

$$
\operatorname{Prob}_{\text {collision }}=\operatorname{Prob}_{\text {co- } \text { channel }}=\frac{1}{\beta}
$$

Co-channel collision corresponds to the case where two communication share the same channel access parameters causing a succession of bit collisions. Co-channel collision is a critical indicator of the quality of the radio access protocol, since a co-channel collision prevent the packet error correction at the receiver.

\subsection{RD-TSOOK protocol}

Like TS-OOK, Rate Division Time Spread On-Off Keying (RD-TSOOK) protocol is a modulation and channel sharing mechanism based on the transmission of femtosecond-long pulses, which are transmitted following an On-Off Keying modulation spread in time. RD-TSOOK is an improved version of TS-OOK protocol, where the transmitter starts by announcing the used $\beta$ before sending the data. The announcement packet is sent over a control channel that uses a specific fixed symbol rate $\beta_{0}$.

$\mathrm{RD}$-TSOOK uses different symbol rates, $\beta$, for the communications. The use of different symbol rates by nanodevices reduces selective interference effect (co-channel collision). Indeed, two communications $C_{1}$ and $C_{2}$ generate a co-channel collision if one of the used symbol rates is a multiple of the other and if the symbols arrival times at the receiver are similar. The following expression demonstrates these two conditions.

$$
\begin{aligned}
& C_{1} \text { is in co-channel with } C_{2} \leftrightarrow \\
& \left(\beta_{C_{1}} \bmod \beta_{C_{2}}=0 \text { AND }\left(\tau_{1}+t 1_{\text {prop }}\right) \quad \bmod T s_{C_{1}}=\left(\tau_{2}+t 2_{\text {prop }}\right) \quad \bmod T s_{C_{2}}\right) \\
& \text { OR } \\
& \left(\beta_{C_{2}} \bmod \beta_{C_{1}}=0 \mathrm{AND}\left(\tau_{1}+t 1_{\text {prop }}\right) \quad \bmod T s_{C_{1}}=\left(\tau_{2}+t 2_{\text {prop }}\right) \quad \bmod T s_{C_{2}}\right)
\end{aligned}
$$

Thus, if a collision happens at a receiver (see Figure 2), the next collision between the same nanonodes could not occur before a period of $\operatorname{LCM}\left(\beta_{C_{1}}, \beta_{C_{2}}\right)$, where LCM is the least common multiple. That is why, in [33, the authors proposed to select the $\beta$ value among a set of coprime values to make that:

$$
\operatorname{LCM}\left(\beta_{C_{1}}, \beta_{C_{2}}\right)=\beta_{C_{1}} \times \beta_{C_{2}}
$$

The following equation demonstrates the RD-TSOOK protocol co-channel collision probability:

$$
\operatorname{Prob}_{\text {co-channel }}=\frac{1}{\beta_{\max }-\beta_{\min }} \times \frac{1}{\beta}
$$


Figure2 2 represents an example of RD-TSOOK where two nanodevices start to transmit to a third common receiver, with different initial transmission times $\tau_{1}$ and $\tau_{2}$. Transmitter 2 is further from the receiver than the first emitter (The different propagation times $t 1_{\text {prop }}$ and $t 2_{\text {prop }}$ ). The upper plot corresponds to the sequence "110100", which is transmitted by the first nanodevice with a symbol rate $T s_{1}$. Likewise, the second trace represents the sequence "100101", transmitted by the second nanodevice with a different symbol rate $T s_{2}$. The signal received at the receiver side is shown on the third trace. In this case, the third symbol of the first nanodevice is overlapped by the second nanodevice's second symbol. Due to the use of different symbol rates, the two nanodevices' consecutive symbols do not overlap.

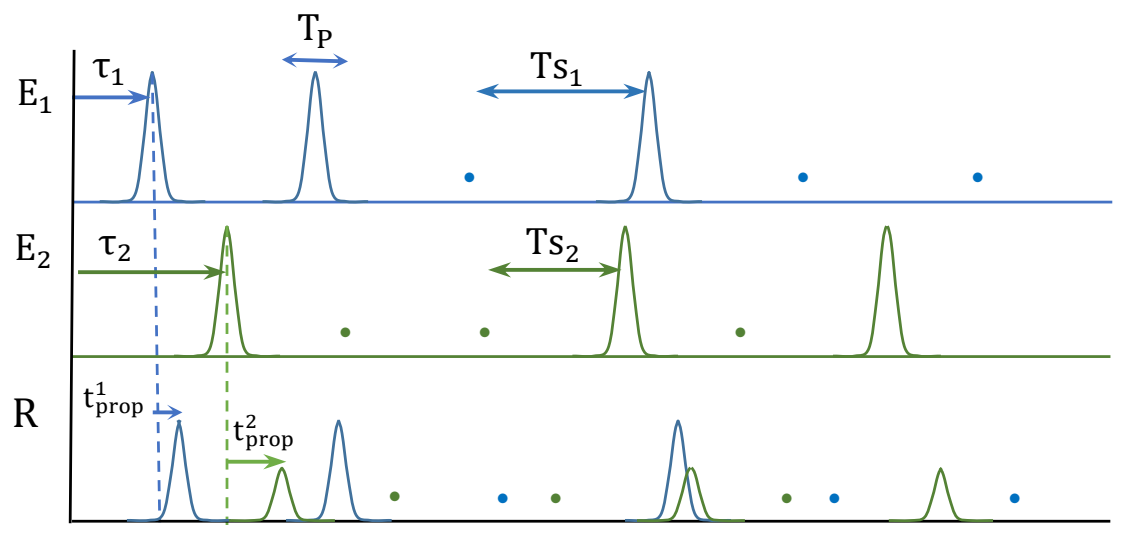

Figure 2: RD-TSOOK modulation: Each nanodevice has its symbol rate different from others.

RD-TSOOK suffers from two major drawbacks. Firstly, the random selection of the symbol rate does not guarantee the uniform use of different $\beta$. Consequently, the nanonodes using a $\beta \approx \beta_{\text {min }}$ benefit from a better throughput than those using a $\beta \approx \beta_{\max }$, which induces an unbalancing between nanodevices.

On the other side, communications with lower symbol rates are more sensitive to collisions when interfering nodes increases. Thus, data retransmission increases, which leads to high energy consumption. Moreover, the use of a high symbol rate when the radio channel is free (low number of active nodes) is not applicable.

Secondly, the protocol presents a complexity for the choice of the range $\left[\beta_{\min }, \beta_{\max }\right]$. RD-TSOOK protocol does not take into account the traffic load to choose the value of $\beta_{\min }$ and $\beta_{\max }$, especially if the channel is not enough busy and $\beta$ chosen tends to $\beta_{\max }$.

\subsection{SRH-TSOOK protocol}

Symbol Rate Hopping Time Spread On-Off Keying (SRH-TSOOK) protocol is based on TS-OOK protocol. The idea is to vary periodically the symbol rate used by communication in a pseudo-random way. This pseudo-random sequence, known by the transmitter and the receiver, is used by the transmitter to change the duration $T_{s}$. In Figure 3 we note that for two consecutive frames $j$ and $j+1$ of the same communication, different $\beta$ are used $\left(\beta_{1} \neq \beta_{1}^{\prime}\right)$. 


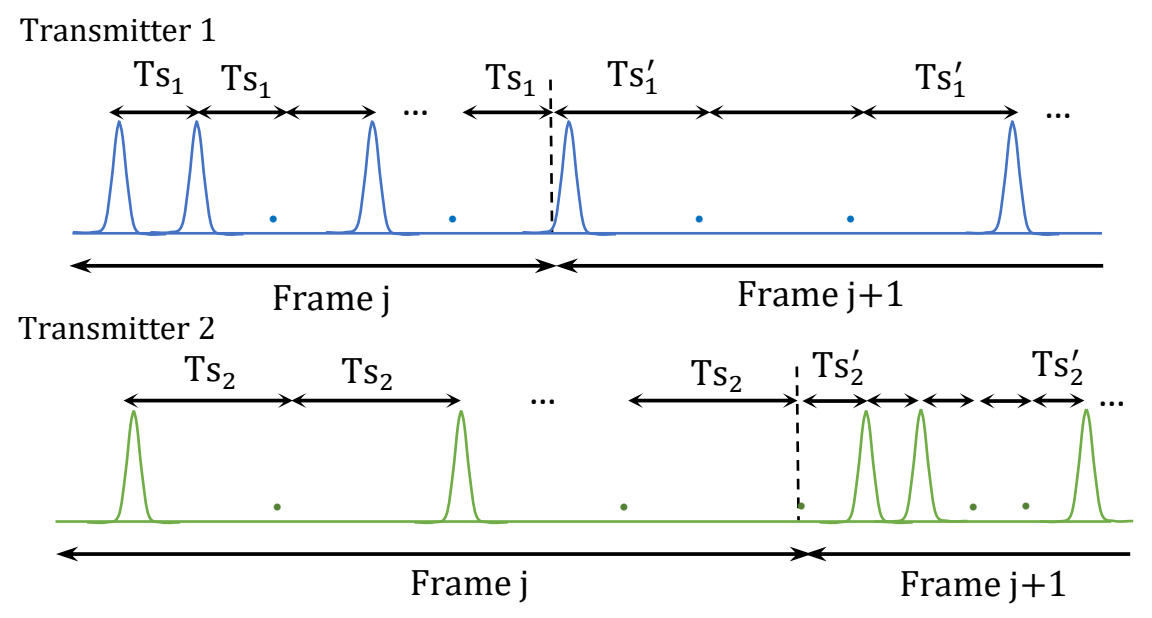

Figure 3: SRH-TSOOK modulation: different symbol rate $\beta$ is used at each frame.

Unlike RD-TSOOK, SRH-TSOOK changes $\beta$ of the communication after a regular number of symbols corresponding to the transmission of a MAC frame. During the establishment of the communication, the transmitter and the receiver agree on an initial value $R_{\text {current }}$. A function $R N D$, generating a random number, is used to change the value of $R_{\text {current }}$ after each frame as follows:

$$
R_{\text {current }}=R N D\left(R_{\text {current }}\right)
$$

The new value of $R_{\text {current }}$ is then recursively calculated without exchange between the receiver and the transmitter. After each frame, a new $\beta$ is computed using the equation:

$$
\beta=\frac{R_{\text {current }} \times\left(\beta_{\max }-\beta_{\min }\right)}{R N D_{\max }}+\beta_{\min }
$$

where $R N D_{\max }$ is the highest value generated by the $R N D$ function.

SRH-TSOOK protocol ensures that the different $\beta$ are used uniformly. Therefore, all active communications ultimately have the same transmission conditions. This approach allows to spread the generated interference over the different communications and gives low similarity radio access conditions to all concurrent communications. Thus, it reduces the probability of co-channel collisions. A co-channel collision occurs when two transmitters $T 1$ and $T 2$ use the same initial $R_{\text {current }}$. This probability is much lower than the probability that two transmitters use the same $\beta$ in RD-TSOOK and TS-OOK protocols. The following expression demonstrates the SRH-TSOOK protocol co-channel collision probability:

$$
\operatorname{Prob}_{\text {co-channel }} \approx \frac{1}{R N D_{\max }} \times \frac{1}{\frac{\left(\beta_{\max }-\beta_{\min }\right)}{2}+\beta_{\min }}
$$

SRH-TSOOK protocol does not take into account the traffic load to choose the value of $\beta_{\max }$ and $\beta_{\min }$. Besides, high values of $\beta$ can be used even when the number of active nanodevices is low. 


\begin{tabular}{|c|c|c|c|}
\hline & \multicolumn{3}{|c|}{ Protocols } \\
\hline Evaluation criteria & TS-OOK & RD-TSOOK & SRH-TSOOK \\
\hline Collisions & $\begin{array}{l}\text { - High collision rate } \\
\text { - The risk of chain } \\
\text { collisions }\end{array}$ & $\begin{array}{l}\text { - Few collisions } \\
\text { - No chain collision }\end{array}$ & $\begin{array}{l}\text { - Few collisions } \\
\text { - No chain collision }\end{array}$ \\
\hline Energy consumption & Very little & Relatively little & Little \\
\hline Balancing Access & Yes & No & Little \\
\hline Complexity & Simple & Simple & Simple \\
\hline $\begin{array}{l}\text { Synchronization } \\
\text { required }\end{array}$ & No & No & No \\
\hline End-to-end flow & $\begin{array}{l}\text { Fixed for all nano- } \\
\text { devices and linked } \\
\text { to the } \beta \text { selected } \\
\text { by the network }\end{array}$ & $\begin{array}{l}\text { Variable from one } \\
\text { nanonode to another } \\
\text { linked to the } \beta \\
\text { chosen by the node }\end{array}$ & $\begin{array}{l}\text { Fairness of the flow } \\
\text { between nodes } \\
\text { according to the } \beta \\
\text { changed each frame }\end{array}$ \\
\hline
\end{tabular}

Table 2: Protocol performance comparison based on probabilistic analysis

\subsection{Preliminary comparison of protocols}

All protocols discussed above have the same characteristics in terms of modulation scheme and communication based on the exchange of very short pulses distributed over time. The difference consists in the channel access technique used to minimize the number of collisions, reduce the co-channel risk and improve radio access fairness.

Selected protocols in our study manipulate the value of $\beta$, to improve their performance. A comparative study of these protocols is given in Table 2 based on probabilistic analysis. This table allows a first evaluation of the performance of these protocols.

\section{Proposed protocol}

Terahertz band communication is the most promising technology to provide high throughput, up to terabits per second $T b / s$. Hence, a wide range of applications that request high resolution accurately benefits from this particularity. Its application in medical diagnostics and treatment has great potential where conventional medical devices are insufficient. In WNN, nanodevices are interconnected via pulse-base communication instead of a high power signal.

In this context, TS-OOK, which is one of the access channel schemes, is based on the exchange of 100 femtosecond-long pulses spread in time. The symbol rate referred to as the interval time between two symbols is fixed and kept the same for all nanodevices in the nanonetwork. Thus, due to the highly deployed nanodevices, the probability of collision increases significantly when multiple nanodevices are transmitting 
simultaneously. If one symbol conflicts with another transmitter's symbol, it will cause conflicts in each next symbol until the end of the packet. These collisions are unacceptable in many applications. On the other hand, channel access balancing is ensured between the nanodevices. All nanodevices have fair channel access.

Regarding to RD-TSOOK technique, each nanodevice randomly generates its symbol rate which is kept during all transmissions. So the collisions phenomena are reduced relatively to TS-OOK; this is due to the low probability to generate identical symbols rate and transmissions starting at synchronized times. Unlike TS-OOK, RD-TSOOK is unbalanced concerning channel access because of the high variation of symbols rate among nanodevices (for example, $\beta_{1}<<\beta_{2}<<\beta_{3} \ldots$ ).

Compared to TS-OOK and RD-TSOOK protocols, SRH-TSOOK generates fewer collisions. This is due to the symbol rate which is renewed at each frame instead of being kept in all transmissions as it does in RD-TSOOK. This symbol rate, regenerated at each frame, makes SRH-TSOOK better balanced than RD-TSOOK, but less than TS-OOK.

Collisions, considered as a significant problem in critical applications, should be greatly reduced or even avoided. In such applications, all interfered data should be retransmitted, resulting then in increased latency. To this end, the proposed SDMA-TSOOK scheme assigns slots randomly to each nanodevice. Our proposal, like the above protocols, does not require any coordination between nanodevices.

In SDMA-TSOOK, nanodevices work in cycles, each cycle of $T s$ duration is composed by $\beta$ slots of $T p$ duration. $\beta$ is a system parameter fixed for all nanonodes like in TS-OOK. The transmitter randomly generates its slot Pslot $\in[0,(\beta-1)]$ to access the channel. For the first time, each nanodevice generates start-time $\tau$ from which it starts generating its slot. This last provides, from equation 10 , the time slot TSlot to access the channel.

$$
\text { TSlot }_{k}=\text { Pslot }_{k} * T p
$$

where $\mathrm{Pslot}_{k}$ is a positive integer number generated by nanodevice $k$ for its symbols' transmission and $T p$ is a time pulse (100 femtosecond-long).

As each nanodevice processes successively two generated values, $\tau$ and Pslot, nanodevices have a low probability of achieving the same time slots. Hence, multiple nanodevices can transmit simultaneously with extremely low interference and collisions. Furthermore, our proposal regulates the access channel where each nanodevice access in a cyclic manner and at a regular interval of time. So, the nanodevice $k$ transmits its first symbol at:

$$
\operatorname{Tr}_{k, 1}=\tau_{k}+\text { TSlot }_{k}
$$

where $k, 1$ denote the nanodevice and the first symbol, respectively.

The rest symbols of the packet are transmitted as follows:

$$
T r_{k, i+1}=T r_{k, i}+T s
$$

Since the nanodevices are asynchronous, although it is low, there is still a weak collision probability even if different time slots are assigned for each nanodevice. For example, two nanodevices 1 and 2 can have 
different start-time $\tau_{1}$ and $\tau_{2}$ such as $\tau_{1}<\tau_{2}$, and different time slot TSlot and $_{1}$ SSlot so $_{2}$ TSlot $_{1}>$ TSlot $_{2}$. These two nanodevices will transmit at the same time if equation 13 is verified. Hence, collision at the receiver will continue to propagate in all the following symbols until the end of the packets.

$$
\tau_{1}+\text { TSlot }_{1}=\tau_{2}+\text { TSlot }_{2}
$$

To overcome such a problem, and minimize co-channel collision probability, a nanodevice regenerates its slots Pslot at each frame, then performs it TSlot according to equation 14, as depicted in Figure 4 .

$$
\begin{array}{rlr}
\operatorname{TSlot}_{k}^{1} & =\operatorname{Pslot}_{k}^{1} * T p & \text { (For the first frame }) \\
\operatorname{TSlot}_{k}^{j+1} & =\left(\left(\beta-\operatorname{Pslot}_{k}^{j}\right)+\operatorname{Pslot}_{k}^{j+1}\right) * T p & \text { (For the rest of frames })
\end{array}
$$

where $\operatorname{Pslot}_{k}^{j}$ and $\operatorname{Pslot}_{k}^{j+1}$ are randomly generated by nanodevice $k$ and $j$ is the number of the frame. Hence, symbols time transmission follows equation 15 :

$$
\begin{array}{rlr}
\operatorname{Tr}_{k, 1}^{1} & =\tau_{k}+\operatorname{TSlot}_{k}^{1} & \text { (For the first symbol of the first frame) } \\
\operatorname{Tr}_{k, i+1}^{1}=\operatorname{Tr}_{k, i}^{1}+T s & \text { (For the rest of symbols of the first frame) } \\
\operatorname{Tr}_{k, i+1}^{j}=\operatorname{Tr}_{k, i}^{j}+T s & \text { (For the symbols of the rest frames) }
\end{array}
$$

This work aims to ensure a fair sharing access channel as well as could efficiently reduce the collision probability to achieve a highly successful packet delivery rate.

A co-channel collision occurs in a frame when two transmitters $T 1$ and $T 2$ use the same initial $\tau$ and the same TSlot as shown in the equation below:

$$
\operatorname{Prob}_{\text {co-channel }}=\frac{1}{\tau} \times \frac{1}{\text { TSlot }}
$$

Due to many factors affecting the performance quality of applications, different criteria should be taken into account, during channel access design, such as generation traffic, deployment, and density of nanodevices in WNN. To this end, a comparison of the channel access protocols seen in section 3 should be done on several scenarios.

\section{Scenarios Implementation}

To verify the effectiveness of Slot Division Multiple Access technique, we compare its performance with those of TS-OOK, RD-TSOOK, and SRH-TSOOK that have been all implemented in BitSimulator 22. The simulation results are evaluated through extensive simulations in which 24 scenarios have been implemented. The different scenarios are discussed below. WNNs are used in different applications, characterized by different topologies of traffic demand, geographical repartition, and density of nanodevices. To this end, we implemented different scenarios with different distributions of nanodevices over the network surface, different WNN densities, and different data traffic profiles. 


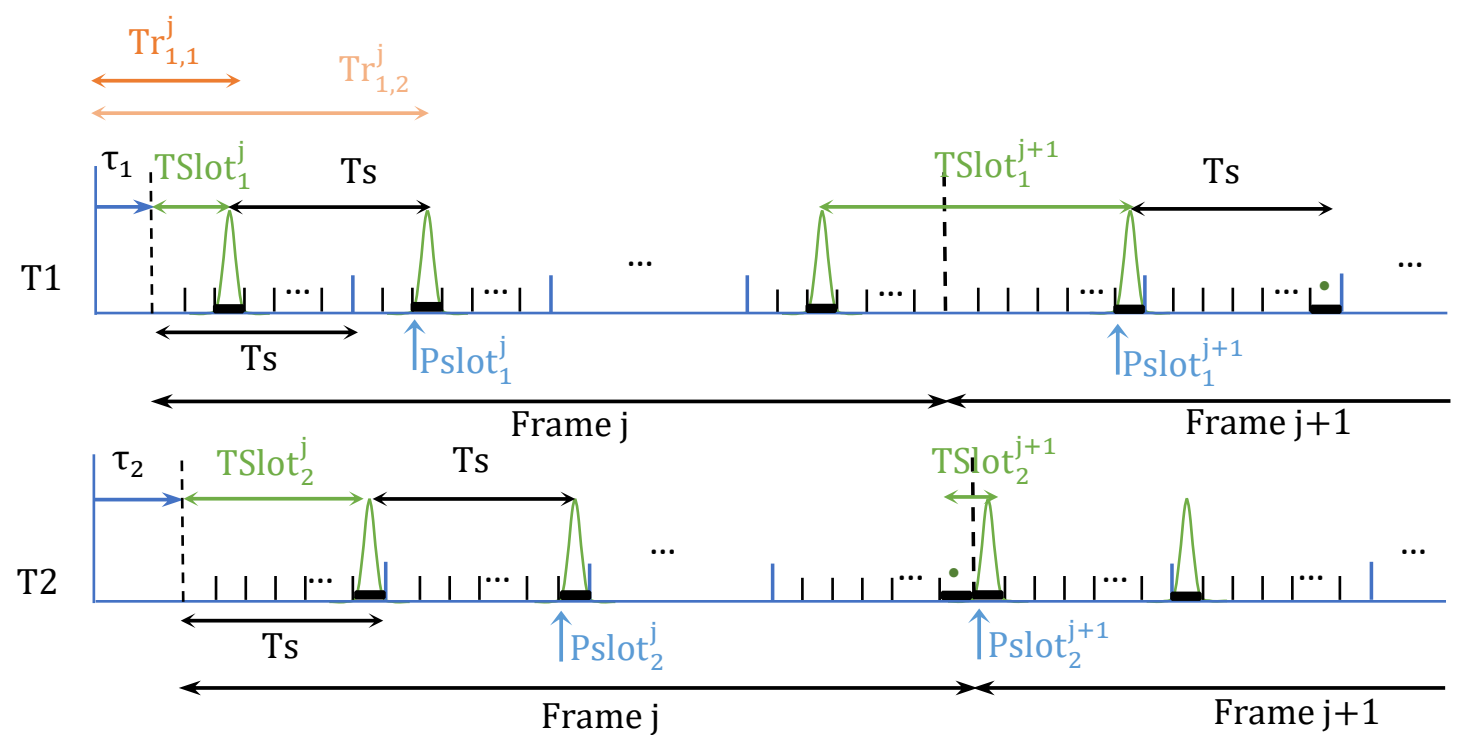

Figure 4: SDMA-TSOOK: different time slot $P$ slot is used at each frame for each node.

\subsection{Nanonodes deployment}

Nanodevices are deployed in varied ways: Uniform distribution, Gaussian distribution, or Gaussian Clusters distribution. Figure 5 presents three distribution's models of the nanodevices on a surface of $4 \mathrm{~mm} * 4 \mathrm{~mm}$.

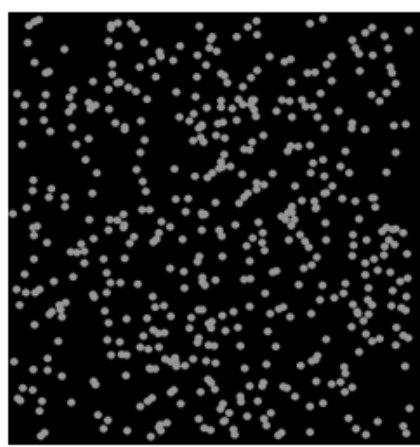

a) Uniform

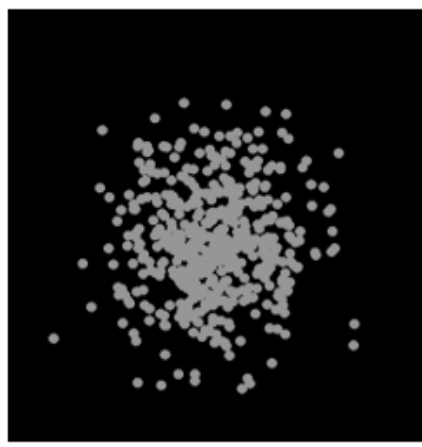

b) Gaussian

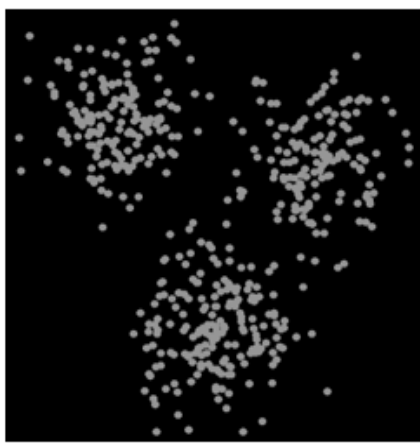

c) Clusters

Figure 5: Different nanonetwork distribution models

\subsubsection{Uniform deployment}

Bettstetter in [36] showed a relationship between nodes transmission range $r_{c}$ and network connectivity as:

$$
r_{c} \geq \sqrt{\frac{-\ln \left(1-p^{1 / n}\right)}{\rho}}
$$

Where $p$ is the possibility that no nanodevice in the network is isolated, $n$ is the total number of nanodevices deployed in the area $A$ satisfying $A<<r_{c}^{2} \pi$ and $\rho=n / A$ is the nanodevice density. 

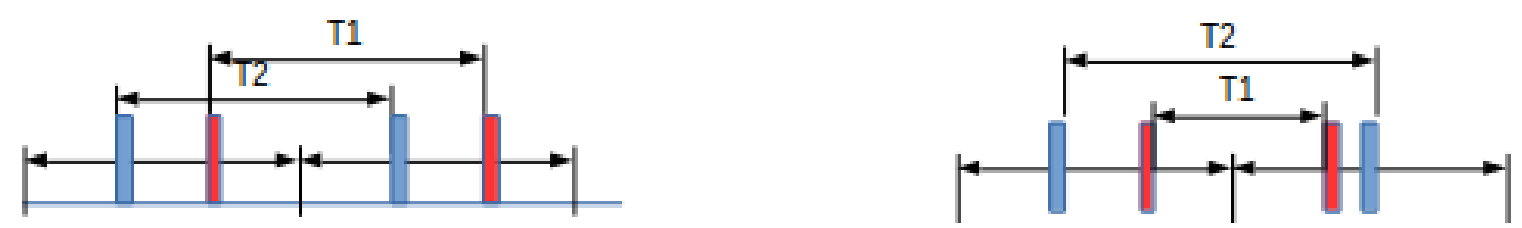

Figure 6: Illustration of two data traffic generation methods

\subsubsection{Gaussian distribution}

In a Gaussian distributed network, the probability that a nanodevice resides at a position with a coordinates $(\mathrm{x}, \mathrm{y})$ relatively to the center of the deployment area $(0,0)$, follows the Gaussian law:

$$
\begin{aligned}
X & \sim \mathcal{N}\left(\mu, \sigma^{2}\right) \\
Y & \sim \mathcal{N}\left(\mu, \sigma^{2}\right) \\
\mu & =N \text { etworkSize } / 2 \\
\sigma & =\text { NetworkSize } / 6
\end{aligned}
$$

where $\mu$ is the mean of the distribution, $\sigma$ is the standard deviation and NetworkSize corresponds to the width of the deployment area (corresponding to a square).

\subsubsection{Gaussian Clusters distribution}

In this distribution, nanodevices deployment corresponds to a set of clusters. Moreover, their positions' coordinates in the cluster follow the law given in equation 19 .

$$
\begin{aligned}
X & \sim \mathcal{N}\left(\mu, \sigma^{2}\right) \\
Y & \sim \mathcal{N}\left(\mu, \sigma^{2}\right) \\
\mu & =\text { num }_{\text {cluster } * \text { NetworkSize } / 4} \\
\sigma & =\text { NetworkSize } / 10
\end{aligned}
$$

where num $_{\text {cluster }} \in\left[1, n b r_{\text {cluster }}\right]$ and each nonodevice chooses its cluster in a random way.

\subsection{Data traffic control}

Other parameters are added to the simulation, monitoring data traffic source-to-destination. Traffic model is a crucial factor in network simulations in order to analyze how the WNN reacts (i.e., congestion) to the traffic conditions (communication arrivals and duration). As can be seen from the analysis of Figure 6 . each transmitter ( 1 and 2 ) sends data packets every $T 1$ and $T 2$ time, respectively. The transmissions can be more or less overlapped according to the communication arrival and traffic amount. Therefore, data traffic behavior is analyzed through simulation using the two primary traffic techniques, CBR and VBR [37. 


\subsubsection{Constant Bit Rate (CBR)}

The CBR technique deals with random fixed delays on the regular data packet transmission. This model is suitable for any communication network having fixed bandwidth permanently available for the entire transmission time. Figure 6 on the left shows this type of traffic where $T 1=T 2$ (i.e., all transmitters have the same transmission cycle).

\subsubsection{Variable Bit Rate (VBR)}

The VBR traffic model uses variable random delays on data packet transmission. We will rely on the Poisson distribution to generate the value of this flow that varies over time. VBR is suitable for the real-time system where data are transmitted at variable flow rates over time. As shown in Figure 6 to the right, each transmitter has a different transmission cycle.

\subsection{Density factor}

As discussed earlier, WNNs are characterized by high density degree that affects their behavior. That is why we simulated the protocols cited in Section 3 with different densities (100, 250, 500, and 750) nanodevices on a surface of $4 \mathrm{~mm} * 4 \mathrm{~mm}$. Figure 7 shows the nanodevices distributions representing different WNN densities.

In the following, all these combined trilogy factors (distribution model, traffic generation method, density) will be examined to assess these protocols' performance. So we get $(3 * 2 * 4)$ nanonetwork scenarios. For each scenario, we implement the four studied channel access protocols.

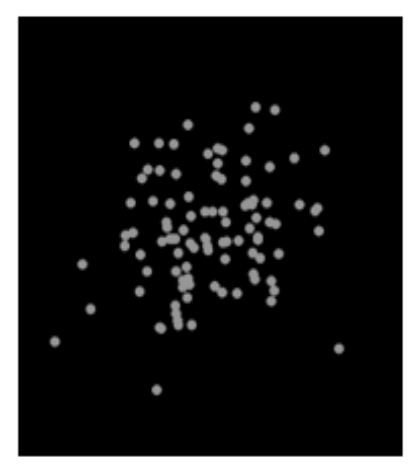

Density 100

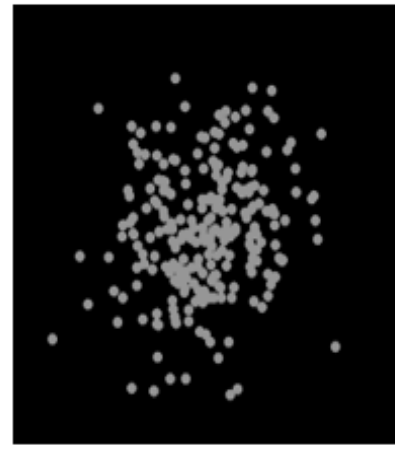

Density 250

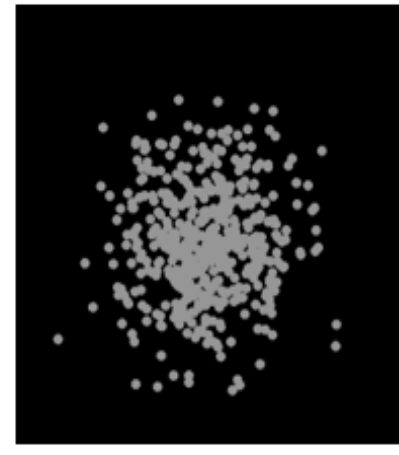

Density 500

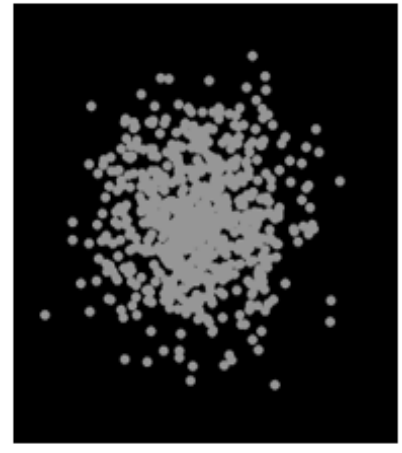

Density 750

Figure 7: Configurations of WNNs with different densities

The protocols implementations are based on the following simulation parameters:

- Routing protocol: Stateless Linear Routing (SLR) protocol [38 is used to transfer the data from the source to the destination nanonodes.

- Each nanonode transmits four packets during the simulation 
- Data packet size: 1000 bits

- Network size: $4 \mathrm{~mm} * 4 \mathrm{~mm}$

- $\beta$ by default equals 3000 for TS-OOK and SDMA-TSOOK while $\beta$ belongs to $[1000,5000]$ for RDTSOOK and SRH-TSOOK.

- Communication range of each nanodevice: $0.5 \mathrm{~mm}$

- Error rate: $10 \%$ of the packet

\section{Performance analysis and comparison}

In this section, through extensive simulations, we exhibit the performance of SDMA-TSOOK, compared to the three discussed protocols, using the 24 scenarios presented in Section 5 (i.e., different densities (100, 250, 500, and 750) with two traffic types (CBR and VBR) and three distributions (Uniform, Gaussian, and Gaussian Clusters)) and then we discuss the results in details. In order to evaluate the performance of the proposal, we used two plausible metrics: number of collisions and transmission success rate.

\subsection{Collision metric}

Figure 8 shows that TS-OOK protocol produced a very high collision number compared to other protocols (RD-TSOOK, SRH-TSOOK, and SDMA-TSOOK). Furthermore, SRH-TSOOK generated about 30\% (resp. $80 \%$ ) of collisions produced in RD-TSOOK with a density of 100 (resp. 750) nanodevices. For instance, SRH-TSOOK generates less than 1030 collisions over the 250 nanodevices, with a Gaussian distribution and VBR traffic, while RD-TSOOK reached 1391 collisions, and TS-OOK reached 4613 collisions.

These results show the effect of density and distribution on the number of collisions in different protocols, where collisions increase over the increasing density. This is due to the high traffic in nanonetwork, and many nanodevices in neighborhood, particularly in Gaussian distribution. For example, with Uniform distribution, 5 (resp. 179) collisions in SRH-TSOOK is recorded with a density of 100 (resp. 250) nanodevices, whereas, in Guassian distribution, the number of collisions reaches 46 (resp. 582) and 23 (resp. 523) with Cluster distribution. The number of generated collisions is much more reduced in SDMA-TSOOK.

\subsection{Success rate metric}

Figure 9 illustrates the effect of the density and distribution scheme on the success rate, i.e., the number of packets having reached their destinations, compared to the total number of transmitted packets.

With increasing density, the preference of SDMA-TSOOK appears on other protocols (SRH-TSOOK, RD-TSOOK, and TSOOK), particularly in the Gaussian distribution where nanodevices are very close. For example, with 750 nanonodes and VBR traffic, SDMA-TSOOK protocol reaches a success rate of $90.3 \%$ with Gauss distribution, and $90.35 \%$ with Cluster distribution; while the success rate is about $84 \%$ for TS-OOK, SRH-TSOOK, RD-TSOOK for the two respective scenarios. 




Figure 8: Comparison of collisions number in different scenarios

From this figure, we conclude that with the Uniform distribution, more packets reach their destinations in RD-TSOOK protocol than other protocols. However, with increasing density, SRH-TSOOK has the best success rate.

\subsection{Discussion of protocols performance}

From the simulation results, we observe that the number of collisions is very low in SDMA-TSOOK due to the used slots' division, where each node chooses a dynamic time slot. Several nanodevices can transmit their data successively one after another. 


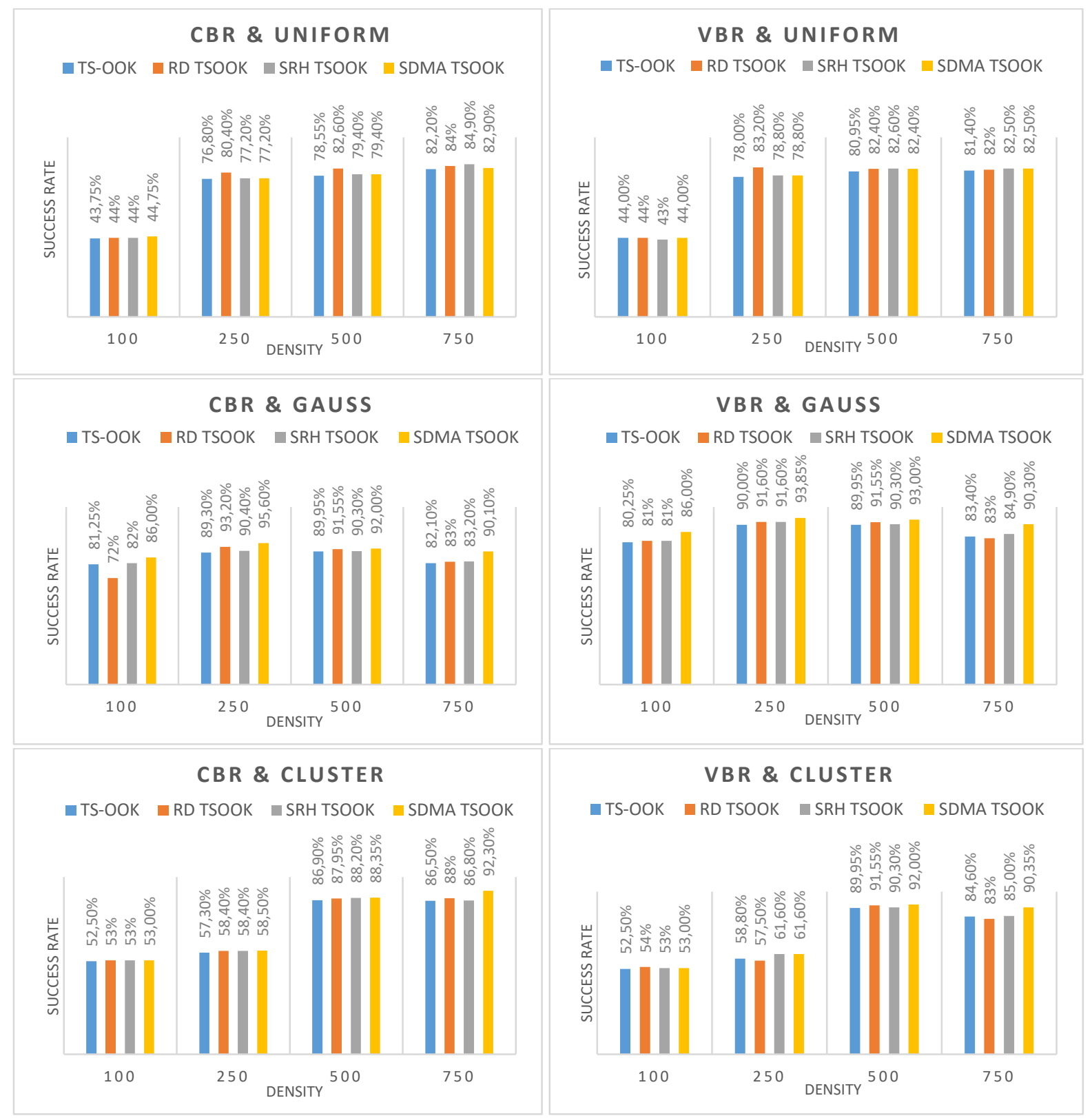

Figure 9: Comparison of success rate in different scenarios

At the receiver, the number of packets accepted is limited (depending on the size of its buffer). Thus, the rest of the arrived packets are ignored (they never reach their final destination), so they will not be considered in the calculation of the success rate, that is why in SDMA-TSOOK, the collision number is negligible, but the success rate does not reach $100 \%$.

According to the results obtained, in all protocols, the success rate is weak under low densities (100 and 250 nanodevices) due to the nanonetwork disconnection (the destination node is unreachable from the source node). This phenomenon is specifically observed in the Uniform distribution, i.e., the nanodevices are distributed over the entire network uniformly. On the other hand, the success rate increases with the 
Gaussian distribution, where the nanonodes are concentrated around the deployment area center.

Also, although the high number of collisions, in TSOOK, RD-TSOOK, and SRH-TSOOK protocols, the success rate is high. This is due to the Stateless Linear Routing (SLR) protocol used in the simulation, which defines linear routing paths among communicating pairs, allowing a considerable degree of parallel transmissions of a packet in the nanonetwork. Thus, a packet collided at an intermediate nanodevice takes another path to reach its destination.

\subsection{Analysis}

All simulation results are summarized in Figure 10. This figure can help to select the best protocol suited according to the application priorities as follows:

- If the application purpose is reducing collisions, the most suitable protocol is SDMA-TSOOK.

- If the application has a dense and concentrated nanonetwork, the SDMA-TSOOK protocol is best suited.

- If the distribution is Uniform with an average density, the RD-TSOOK protocol is more favored. However, RD-TSOOK leads to variable throughput from a communication to another.

- With a high density (over 500 nanodevices) and Uniform distribution, SRH-TSOOK protocol is preferable.

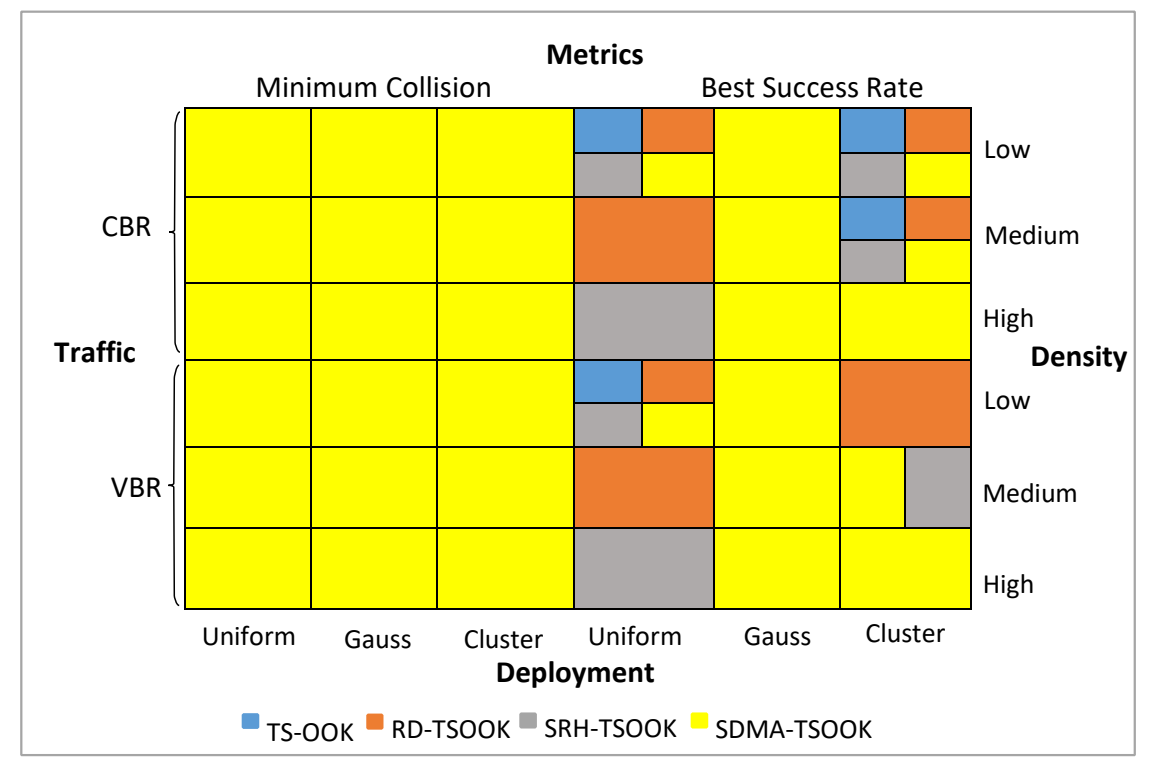

Figure 10: Scenarios \& protocols appropriate for application types

According to this comparative study, several observations can be made. First, it turns out that nanonetworks have behaviors that only appear during the simulation. Therefore, a probabilistic study of protocols is 
not sufficient to validate their performance. Second, each protocol behaves differently depending on the application type. Third, choosing the best protocol depends on the deployment area and the objectives targeted by the application. SDMA-TSOOK is more suitable for applications such as software-defined meta-materials 39] and controllable robotic materials [40] which require high densely nanonetworks.

Finally, we conclude in this study that SDMA-TSOOK differs from other protocols by reducing collisions number in different performed simulations. Moreover, it ensures channel access balancing between nanodevices. RD-TSOOK shows a better success rate with a low density and a Uniform distribution. However, with a high density, SRH-TSOOK protocol is preferred.

TSOOK protocol ensures a balance among nanodevices in the network with a very high number of collisions and a low success rate compared to other protocols. Success rate is higher in the Gaussian distribution than in other ones, which is due to the huge number of neighborhoods.

SDMA-TSOOK performances could be improved by adjusting the transmission cycle, represented by $\beta$. Figure 11 shows the results obtained by simulating 750 nanodevices network with time slots $\in[0,(\beta-1)]$ where $\beta$ has the values 750/1000/2000/3000. In each graph, three metrics (collision, success rate, and pointto-point delay) are considered. The average point-to-point delay is the average time spent by a packet from its source to its destination (given in nanoseconds).

Note that the success rate for different $\beta$ values remains almost the same, while $\beta$ is inversely proportional to the number of collisions but proportional to point-to-point delay.

\section{Conclusion}

In nanonetworks, the need for an appropriate channel sharing scheme raises a serious challenge for efficiently allowing concurrent channel accesses.

First, we provided an exhaustive critical review of channel access approaches based on femtosecond-Long pulse modulation, particularly TS-OOK, RD-TSOOK, and SRH-TSOOK. This study investigated the drawbacks of each approach. Then, we proposed a Slot Division Multiple Access approach called SDMA-TSOOK to overcome these weaknesses. SDMA-TSOOK is inspired by Time Division Multiple Access technique (TDMA). Each transmitter asynchronously and independently allocates time slot used to send its symbols. To prevent the effect of co-channel collision the time slot is regularly changed, leading to highly mitigating the collisions.

We evaluated the performance of SDMA-TSOOK, compared to those reviewed approaches, in terms of channel access balancing, collisions, and success rate. As a result, our proposal protocol presents a good trade-off between the reviewed approaches. It ensured fairness between nanodevices and significantly reduced collisions while keeping the success rate. 

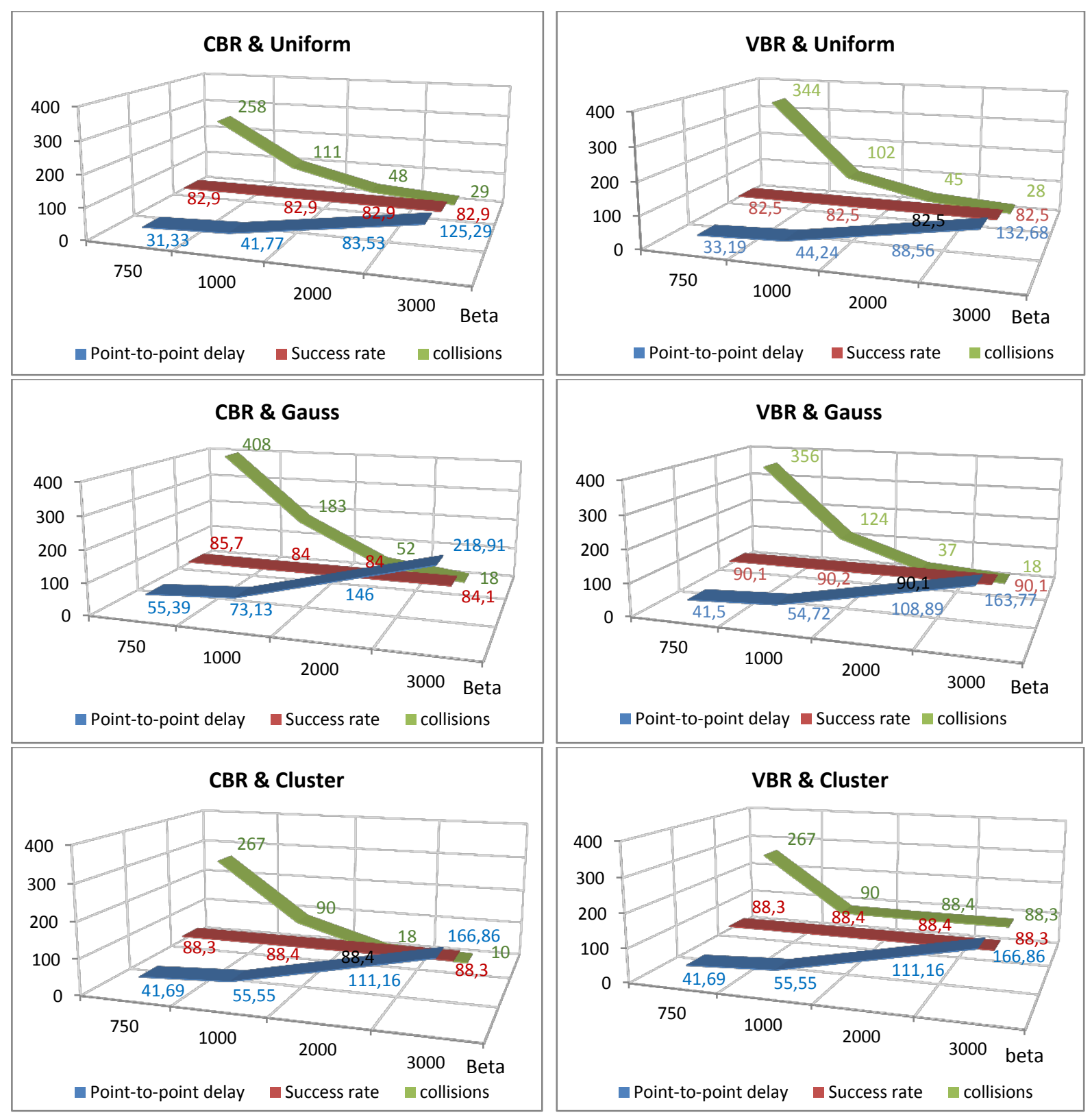

Figure 11: SDMA-TSOOK performance under beta and slots sequence

\section{References}

[1] I. F. Akyildiz, F. Brunetti, C. Blázquez, Nanonetworks: A new communication paradigm, Computer Networks 52 (12) (2008) 2260-2279. doi:10.1016/j.comnet.2008.04.001.

[2] B. Atakan, O. B. Akan, S. Balasubramaniam, Body area nanonetworks with molecular communications in nanomedicine, IEEE Communications Magazine 50 (1) (2012) 28-34. doi:10.1109/MCOM.2012. 6122529 .

[3] H. Mabed, J. Bourgeois, Towards programmable material: Flexible distributed algorithm for modular 
robots shape-shifting, in: 2014 IEEE/ASME International Conference on Advanced Intelligent Mechatronics, IEEE, 2014, pp. 408-414. doi:10.1109/AIM.2014.6878113.

[4] B. Lebental, B. Ghaddab, F. Michelis, Nanosensors for embedded monitoring of construction materials: The "2d conformable" route, in: Nanotechnology in Construction, Springer, 2015, pp. 383-388.

[5] N. Boillot, D. Dhoutaut, J. Bourgeois, Large scale mems robots cooperative map building based on realistic simulation of nano-wireless communications, Nano Communication Networks 6 (2) (2015) 5173.

[6] I. F. Akyildiz, J. M. Jornet, Electromagnetic wireless nanosensor networks, Nano Communication Networks 1 (1) (2010) 3-19. doi:10.1016/j.nancom.2010.04.001.

[7] F. Lemic, S. Abadal, W. Tavernier, P. Stroobant, D. Colle, E. Alarcón, J. Marquez-Barja, J. Famaey, Survey on terahertz nanocommunication and networking: A top-down perspective, arXiv preprint arXiv:1909.05703.

[8] T. Kürner, Towards future thz communications systems, Terahertz science and technology 5 (1) (2012) $11-17$.

[9] D. Correas-Serrano, J. S. Gomez-Diaz, Graphene-based antennas for terahertz systems: A review, arXiv preprint arXiv:1704.00371.

[10] J. M. Jornet, I. F. Akyildiz, Graphene-based nano-antennas for electromagnetic nanocommunications in the terahertz band, in: Antennas and Propagation (EuCAP), 2010 Proceedings of the Fourth European Conference on, IEEE, 2010, pp. 1-5.

[11] I. F. Akyildiz, J. M. Jornet, C. Han, Terahertz band: Next frontier for wireless communications, Physical Communication 12 (2014) 16-32. doi:10.1016/j.phycom.2014.01.006.

[12] E. Zarepour, M. Hassan, C. T. Chou, A. A. Adesina, Frequency hopping strategies for improving terahertz sensor network performance over composition varying channels, in: Proceeding of IEEE International Symposium on a World of Wireless, Mobile and Multimedia Networks 2014, IEEE, 2014, pp. 1-9. doi:10.1109/WoWMoM.2014.6918973.

[13] V. Petrov, A. Pyattaev, D. Moltchanov, Y. Koucheryavy, Terahertz band communications: Applications, research challenges, and standardization activities, in: 2016 8th International Congress on Ultra Modern Telecommunications and Control Systems and Workshops (ICUMT), IEEE, 2016, pp. 183-190.

[14] Z. Hossain, J. M. Jornet, Hierarchical bandwidth modulation for ultra-broadband terahertz communications, in: ICC 2019-2019 IEEE International Conference on Communications (ICC), IEEE, 2019, pp. 1-7. 
[15] G. C. Bjorklund, M. Levenson, W. Lenth, C. Ortiz, Frequency modulation (fm) spectroscopy, Applied Physics B 32 (3) (1983) 145-152.

[16] P. Laurent, Exact and approximate construction of digital phase modulations by superposition of amplitude modulated pulses (amp), IEEE transactions on communications 34 (2) (1986) 150-160.

[17] T. Aulin, N. Rydbeck, C.-E. Sundberg, Continuous phase modulation-part ii: Partial response signaling, IEEE Transactions on Communications 29 (3) (1981) 210-225.

[18] M. Saad, F. Bader, J. Palicot, A. C. Al Ghouwayel, H. Hijazi, Single carrier with index modulation for low power terabit systems, in: 2019 IEEE Wireless Communications and Networking Conference (WCNC), IEEE, 2019, pp. 1-7.

[19] I. Llatser, A. Cabellos-Aparicio, M. Pierobon, E. Alarcón, Detection techniques for diffusion-based molecular communication, IEEE Journal on Selected Areas in Communications 31 (12) (2013) 726-734. doi:10.1109/JSAC.2013.SUP2.1213005.

[20] A. Aijaz, A.-H. Aghvami, Error performance of diffusion-based molecular communication using pulsebased modulation, IEEE transactions on nanobioscience 14 (1) (2014) 146-151.

[21] J. M. Jornet, I. F. Akyildiz, Channel modeling and capacity analysis for electromagnetic wireless nanonetworks in the terahertz band, IEEE Transactions on Wireless Communications 10 (10) (2011) 32113221. doi:10.1109/TWC.2011.081011.100545.

[22] D. Dhoutaut, T. Arrabal, E. Dedu, Bit simulator, an electromagnetic nanonetworks simulator, in: Proceedings of the 5th ACM International Conference on Nanoscale Computing and Communication, 2018, pp. 1-6. doi:10.1145/3233188.3233205.

[23] P. Singh, B.-W. Kim, S.-Y. Jung, Th-ppm with non-coherent detection for multiple access in electromagnetic wireless nanocommunications, Nano Communication Networks 17 (2018) 1-13.

[24] A. Moldovan, S. Kisseleff, I. F. Akyildiz, W. H. Gerstacker, Data rate maximization for terahertz communication systems using finite alphabets, in: 2016 IEEE International Conference on Communications (ICC), IEEE, 2016, pp. 1-7.

[25] L. Aliouat, H. Mabed, J. Bourgeois, 2.5 layer protocol for traffic regulation in ultra-dense nanonetwork, in: International Conference on Ad-Hoc Networks and Wireless, Springer, 2019, pp. 330-340.

[26] J. M. Jornet, I. F. Akyildiz, Low-weight channel coding for interference mitigation in electromagnetic nanonetworks in the terahertz band, in: 2011 IEEE international conference on communications (ICC), IEEE, 2011, pp. 1-6. doi:10.1109/icc.2011.5962987. 
[27] P. Singh, B.-W. Kim, S.-Y. Jung, Th-ppm with non-coherent detection for multiple access in electromagnetic wireless nanocommunications, Nano Communication Networks 17 (2018) 1-13. doi: $10.1016 / j$.nancom.2018.05.001.

[28] M. Kocaoglu, O. B. Akan, Minimum energy channel codes for nanoscale wireless communications, IEEE Transactions on Wireless Communications 12 (4) (2013) 1492-1500. doi:10.1109/TWC.2013.022113. 2190.

[29] K. Chi, Y.-h. Zhu, X. Jiang, X. Tian, Optimal coding for transmission energy minimization in wireless nanosensor networks, Nano Communication Networks 4 (3) (2013) 120-130. doi:10.1016/j.nancom. 2013.07.001.

[30] P. Singh, B. W. Kim, S.-Y. Jung, Ds-ook for terahertz band nanonetworks, National Academy Science Letters (2020) 1-4doi:10.1007/s40009-020-00955-7.

[31] A. P. Shrestha, S.-J. Yoo, H. J. Choi, K. S. Kwak, Enhanced rate division multiple access for electromagnetic nanonetworks, IEEE Sensors Journal 16 (19) (2016) 7287-7296.

[32] J. M. Jornet, I. F. Akyildiz, Femtosecond-long pulse-based modulation for terahertz band communication in nanonetworks, IEEE Transactions on Communications 62 (5) (2014) 1742-1754. doi: 10.1109/TCOMM.2014.033014.130403.

[33] J. M. Jornet, J. C. Pujol, J. S. Pareta, Phlame: A physical layer aware mac protocol for electromagnetic nanonetworks in the terahertz band, Nano Communication Networks 3 (1) (2012) 74-81. doi:10.1109/ INFCOMW. 2011.5928852.

[34] H. Mabed, Enhanced spread in time on-off keying technique for dense terahertz nanonetworks, in: 2017 IEEE Symposium on Computers and Communications (ISCC), IEEE, 2017, pp. 710-716. doi: 10.1109/ISCC.2017.8024611.

[35] A. K. Vavouris, F. D. Dervisi, V. K. Papanikolaou, G. K. Karagiannidis, An energy efficient modulation scheme for body-centric nano-communications in the thz band, in: 2018 7th International Conference on Modern Circuits and Systems Technologies (MOCAST), IEEE, 2018, pp. 1-4. doi:10.1109/MOCAST. 2018.8376563 .

[36] C. Bettstetter, On the minimum node degree and connectivity of a wireless multihop network, in: Proceedings of the 3rd ACM international symposium on Mobile ad hoc networking \& computing, 2002, pp. 80-91. doi:doi.org/10.1145/513800.513811.

[37] G. D. Kondylis, S. V. Krishnamurthy, S. K. Dao, G. J. Pottie, Multicasting sustained cbr and vbr traffic in wireless ad-hoc networks, in: 2000 IEEE International Conference on Communications. ICC 2000. Global Convergence Through Communications. Conference Record, Vol. 1, IEEE, 2000, pp. 543-549. doi:10.1109/ICC.2000.853377. 
[38] A. Tsioliaridou, C. Liaskos, E. Dedu, S. Ioannidis, Stateless linear-path routing for 3d nanonetworks, in: Proceedings of the 3rd ACM International Conference on Nanoscale Computing and Communication, 2016, pp. 1-6. doi:10.1145/2967446.2967451.

[39] S. Abadal, C. Liaskos, A. Tsioliaridou, S. Ioannidis, A. Pitsillides, J. Solé-Pareta, E. Alarcón, A. Cabellos-Aparicio, Computing and communications for the software-defined metamaterial paradigm: A context analysis, IEEE access 5 (2017) 6225-6235.

[40] N. Correll, P. Dutta, R. Han, K. Pister, Wireless robotic materials, in: Proceedings of the 15th ACM Conference on Embedded Network Sensor Systems, 2017, pp. 1-6.

[41] A. C. Tasolamprou, A. D. Koulouklidis, C. Daskalaki, C. P. Mavidis, G. Kenanakis, G. Deligeorgis, Z. Viskadourakis, P. Kuzhir, S. Tzortzakis, M. Kafesaki, et al., Experimental demonstration of ultrafast thz modulation in a graphene-based thin film absorber through negative photoinduced conductivity, ACS photonics 6 (3) (2019) 720-727.

[42] J. Feng, X. Zhao, Performance analysis of ook-based fso systems in gamma-gamma turbulence with imprecise channel models, Optics Communications 402 (2017) 340-348. 